\title{
Patrimonio cultural, investigación e innovación en el marco del Horizonte 2020
}

En el Programa Marco de Investigación e Innovación Horizon 2020 de la Unión Europea, que estará vigente de 2014 a 2020, las humanidades y ciencias sociales se incorporan como elemento esencial de las actividades necesarias para abordar los diferentes retos planteados. Frente a reiteradas demandas, el patrimonio cultural aparece como transversal con el objeto de ser herramienta para el desarrollo y no un fin en sí mismo. La clave -en palabras de Ramón Flecha, Catedrático de Sociología de la Universidad de Barcelona- estaría en "investigar con y para la sociedad, buscando la socialización, lo que implica pasar previamente por el impacto científico y político".

M. ${ }^{a}$ Rosa Tera Saavedra | coordinadora del grupo de trabajo de Patrimonio del Cluster para la Construcción Eficiente y Hábitat (AEICE)

URL de la contribución <www.iaph.es/revistaph/index.php/revistaph/article/view/3520>

Con el objeto de fomentar la mayor presencia de profesionales y propuestas exitosas relacionadas con patrimonio cultural en las diferentes convocatorias del Programa Horizonte 2020, el 31 de marzo de 2014, se realizó en el Instituto de Patrimonio Cultural de España (IPCE) la jornada Horizonte 2020 y Patrimonio Cultural: Investigación e Innovación, promovida por la asociación Ars Civilis.

En ella, diferentes técnicos presentaron aspectos del programa que pueden vincularse con patrimonio cultural y diferentes ponentes expusieron sus experiencias concretas relacionadas con convocatorias europeas y patrimonio.

También fue presentado el programa de investigación JPI Cultural Heritage and Global Change ${ }^{1}$ que surge de la visión conjunta de la estrategia europea Strategic Research Agenda (SRA), que se preselecciona a través del Punto de Contacto Nacional de España, y que puede tener sinergias con fondos estructurales. Como ejemplo de convocatoria se mencionó la propuesta Heritage Plus, centrada principalmente en la investigación del patrimonio cultural tangible, aunque no excluye los aspectos interrelacionados del patrimonio inmaterial y digital.

Las diferentes presentaciones expuestas en la jornada pueden consultarse en la página de la asociación ${ }^{2}$ y en formato de resumen (TERA SAAVEDRA, 2014).

\section{Investigación que genere empleo}

Horizonte 2020 es el nombre del Programa Marco de Investigación e Innovación que, aprobado por el Parlamento Europeo y el Consejo de Europa, estará vigente desde este año 2014 hasta $2020^{3}$.

Es uno de los programas que se han desarrollado con el objeto de dar respuesta a los objetivos de la estrategia Europa 2020. Surge como propuesta para reactivar la economía europea ante el cambio de contexto económico provocado desde la crisis financiera de 2008.

Las líneas estratégicas que utiliza están basadas en la investigación e innovación como herramientas capaces de aportar conocimiento que sirve para crear productos y servicios que dan respuesta a retos sociales relacionados con un desarrollo sostenible, integrador, que aporta crecimiento y empleo.

Como novedad frente a la estrategia desarrollada en anteriores programas marco, y con los objetivos de fomentar un crecimiento inteligente, sostenible e integrador que aporte ideas, crecimiento y empleo, el programa Horizon 2020 pretende:

> simplificar el acceso, normas y control para optimizar la gestión; 
> concebir la innovación como un conjunto de valores que es capaz de relacionar productos, procesos y retos sociales;

> reforzar la participación de la PYME;

> fomentar la cooperación internacional entre los países de la Unión y economías emergentes;

> buscar la excelencia a través de la participación de universidades y centros de investigación;

> lograr la transferencia social a través de la promoción y acceso abierto a toda la información de los proyectos financiados por el programa;

$>$ crear un mercado único de conocimiento que permita la cooperación transfronteriza a través de la creación del Espacio Europeo de Investigación (EEI).

Las convocatorias se van convocando cíclicamente a través de diferentes llamadas y responden a un principio de cofinanciación por parte de fondos europeos que puede llegar hasta al $70 \%$.

En este amplio marco el patrimonio cultural es transversal, puede tener cabida en numerosas convocatorias. Las humanidades y ciencias sociales se pueden incorporar como elemento esencial de las actividades necesarias para abordar diferentes retos planteados en el programa.

\section{Investigación en patrimonio cultural}

Diferentes aspectos de investigación, que son necesarios abordar en relación con el patrimonio cultural, pueden incluirse en las acciones indirectas, o partes, que existen en el Horizon 2020. Las propuestas de innovación incluyen los ámbitos tecnológicos, sociales y relacionados con el sector público, siendo su fin último el adaptar y transformar los resultados en productos y servicios útiles para la sociedad.

Por ejemplo, la parte I (ciencia excelente) atañe a diferentes investigadores, entre los que se pueden incluir

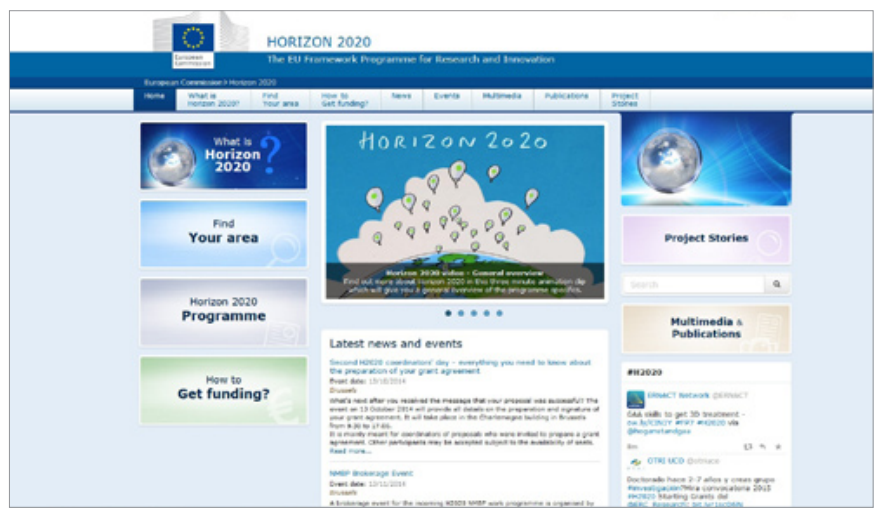

http://ec.europa.eu/programmes/horizon2020/

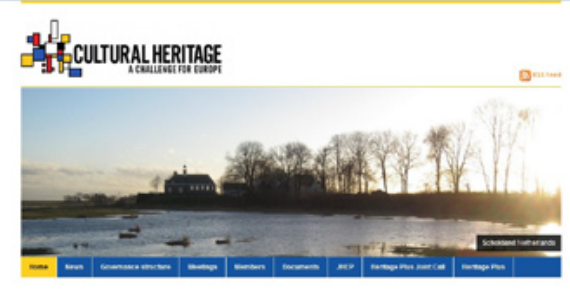

Welcome to JPI on Cultural Heritage

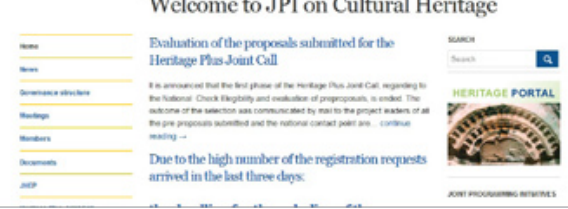

http://www.jpi-culturalheritage.eu/

los relacionados con el patrimonio cultural. Incluye las acciones Marie Sklodowska-Curie (MSCA) a través del programa de movilidad de excelencia investigadora y las acciones incluidas en el European Research Council (ERC).

La parte II (liderazgo industrial) puede relacionarse con numerosas demandas que reclama el patrimonio. Incluirá propuestas que den respuesta a aspectos como el liderazgo en tecnologías industriales y capacitación (LEIT) o la innovación en PYMES, incluyendo acciones relacionadas con el liderazgo industrial, la nanotecnología, creación de nuevos materiales y productos o innovación en la producción, temas de estandarización, planes de negocio y comercialización basándose en pruebas que ya estén validadas en laboratorio a nivel real. En el ámbito patrimonial podrían tener cabida, por ejemplo: 
> el desarrollo de tecnologías de la información y comunicación para la formación e interacción relacionados con educación patrimonial;

$>$ el acceso a contenidos para garantizar el conocimiento y la transferencia de los valores que representa el patrimonio;

> la creación de sistemas inteligentes para gestionar información que puede estar relacionada con conservación preventiva o bases de datos de acceso;

> el análisis estadístico o desarrollo de tecnologías informáticas visuales que sirvan para evaluar cómo el patrimonio puede generar riqueza;

$>$ o el desarrollo de tecnologías para crear nuevas oportunidades empresariales en los que se pueden incluir nuevos materiales para la conservación de elementos con valor histórico o cultural que, por ejemplo, puedan estar relacionados con la nano-tecnología.

La parte III (retos sociales) presenta diferentes desafíos, en los que existe libertad para proponer soluciones. Si entendemos el patrimonio como bien público, cuya preservación en un futuro es bastante probable que dependa de su capacidad para generar riqueza, quizás este apartado pueda servir para innovar en planteamientos de gestión basados en una toma de decisiones eficaz en la que se impliquen compromiso público y social a través de la participación ciudadana ${ }^{4}$.

En el reto 6 (Europa en un mundo cambiante-Sociedades inclusivas, innovadoras y reflexivas) de esta parte III, el patrimonio cultural aparece explícitamente en el apartado C (Sociedades reflexivas: patrimonio cultural e identidad europea) dado que, al ser el resultado de múltiples creencias y sistemas culturales, es nexo integrador para la construcción de sociedades inclusivas, innovadoras y seguras que puede servir para construir una sociedad europea basada en la integración y con capacidad de superación, así como estimular visiones modernas y futuros usos de su pasado ${ }^{5}$. Los temas de esta convocatoria en el 2014 se han centrado en:
$>$ patrimonio cultural/formación de la identidad/formación del legado intelectual, artístico, creativo e histórico. Consorcio ERA-net formado por entidades financiadoras de investigación, como ministerios o agencias, cuyo objetivo es sacar convocatorias conjuntas para la comunidad investigadora;

> tecnologías digitales: modelización/análisis/comprensión y conservación del patrimonio cultural europeo a través de la modelización avanzada 3D para acceder y entender los activos culturales europeos.

En el Horizon 2020 la respuesta al "para qué" ha quedado claramente establecida. No estamos hablando solo de investigación e innovación. Desde mi especial interés por el desarrollo, que creo puede llegar a generar el patrimonio, quiero hacer mención a una idea expresada en la jornada que reafirma mi creencia de que el patrimonio ha de ser considerado como un recurso a través del cual se han de catalizar procesos sociales, culturales y económicos. Tomando las palabras del ponente Ramón Flecha, Catedrático de Sociología de la Universidad de Barcelona y Director del Proyecto IMPACT: "la clave del nuevo programa marco es investigar con y para la sociedad, buscando la socialización, lo que implica pasar previamente por el impacto científico y político". Para ello, los procesos necesarios son: publicación abierta, difusión, transferencia, impacto social y replicabilidad. Intentemos aplicarlos a través del patrimonio. 
1. Cultural Heritage a challenge for Europe <http://www. jpi-culturalheritage.eu/> [Consulta: 05/07/2014]

2. Ars Civilis <http://www.arscivilis.org/es/horizonte2020-y-patrimonio-cultural-investigacion-e-innovacion/> [Consulta: 05/07/2014]

3. European Commission Horizon2020<http://ec.europa. eu/programmes/horizon2020/> [Consulta: 05/07/2014]. Información en español: <http://www.eshorizonte2020. es/> [Consulta: 05/07/2014]

4. Parte III. Retos Sociales; punto 5. Acción por el clima, eficiencia de los recursos y materias primas; apartado 5.2. Gestión sostenible de los recursos naturales y los ecosistemas; subapartado 5.2.2. Proporcionar conocimientos y herramientas que propicien una toma de decisiones eficaz y un compromiso público.

5. Parte III. Retos sociales; punto 6 Sociedades inclusivas, innovadoras y seguras.

\section{Webs y redes sociales}

$>$ Linkedin: https://www.linkedin.com/in/rosatera

$>$ Twiter: https://twitter.com/ROSATERA

$>$ Facebook: https://www.facebook.com/batea.patrimonio

> Blog: http://rosatera.blogspot.com.es/

> Web: http://www.bateaproyecto.com/

\section{BIBLIOGRAFÍA}

- COMUNICACIóN (2010) 2020 de la Comisión Europea, por la que se presenta Europa 2020: Una Estrategia para un Crecimiento Inteligente, Sostenible, e Integrador, de 3 de marzo de 2010. En EUR-Lex <http://eur-lex.europa.eu/legal-content/ ES/TXT/?uri=CELEX:52010DC2020> [Consulta: 05/07/2014]

- RECOMENDACIÓN (UE) n. ${ }^{\circ}$ 410/2010 del Consejo, de 13 de julio de 2010, sobre directrices generales para las políticas económicas de los Estados miembros y de la Unión. Diario Oficial de la Unión Europea, n. ${ }^{\circ} 191$, de 23 de julio de 2010 <http://eur-lex.europa.eu/legal-content/ES/TXT/ ?uri=CELEX:32010H0410> [Consulta: 05/07/2014]

- REGLAMENTO (UE) n. ${ }^{\circ} 1290 / 2013$ del Parlamento Europeo y del Consejo, de 11 de diciembre de 2013, por el que se establecen las normas de participación y difusión aplicables a Horizonte 2020 Programa Marco de Investigación e Innovación (2014-2020). Diario Oficial de la Unión Europea, $\mathrm{n}^{\circ} 347$, de 20 de diciembre de 2013 <http://ec.europa.eu/research/ participants/data/ref/h2020/legal_basis/rules_participation/ h2020-rules-participation_es.pdf> [Consulta: 05/07/2014]

- TERA SAAVEDRA, M. R. (2014) Horizonte ampliado [resumen Jornada Horizonte2020 y Patrimonio Cultural: Investigación e Innovación]. En http://rosatera.blogspot.com. es/ [Blog de Rosa Tera] <http://es.scribd.com/doc/216569880/ Resumen-de-la-Jornada-ARS-Civilis-IPCE> [Consulta: 05/07/ 2014] 